\title{
Death Toll of COVID-19 on Asian Americans: Disparities Revealed
}

\author{
Brandon W. Yan, B.A. ${ }^{1,2}$ (D) Andrea L. Hwang, B.S. ${ }^{2}$, Fiona Ng, B.S., M.P.H. ${ }^{1,2}$, \\ Janet N. Chu, M.D., M.P.H. ${ }^{2,3}$, Janice Y. Tsoh, Ph.D., ${ }^{2,4}$ and Tung T. Nguyen, M.D.2,3
}

${ }^{1}$ School of Medicine, University of California San Francisco, 513 Parnassus Ave., Suite S-214, San Francisco, CA, USA; ${ }^{2}$ Asian American Research Center on Health (ARCH), San Francisco, CA, USA; ${ }^{3}$ Division of General Internal Medicine, University of California San Francisco School of Medicine, San Francisco, CA, USA; 'Department of Psychiatry and Behavioral Sciences, University of California San Francisco School of Medicine, San Francisco, CA, USA.

The coronavirus disease 2019 (COVID-19) pandemic has underscored the structural inequities facing communities of color and its consequences in lives lost. However, little is known about the COVID-related disparities facing Asian Americans amidst the heightened racism and violence against this community. We analyze the mortality toll of COVID-19 on Asian Americans using multiple measures. In 2020, one in seven Asian American deaths was attributable to COVID-19. We find that while Asian Americans make up a small proportion of COVID-19 deaths in the USA, they experience significantly higher excess all-cause mortality (3.1 times higher), case fatality rate (as high as $53 \%$ higher), and percentage of deaths attributed to COVID-19 (2.1 times higher) compared to non-Hispanic Whites. Mounting evidence suggest that disproportionately low testing rates, greater disease severity at care presentation, socioeconomic factors, and racial discrimination contribute to the observed disparities. Improving data reporting and uniformly confronting racism are key components to addressing health inequities facing communities of color.

KEY WORDS: Asian American; mortality; COVID; health disparities; racism.

$\mathrm{J}$ Gen Intern Med 36(11):3545-9

DOI: $10.1007 / \mathrm{s} 11606-021-07003-0$

(C) Society of General Internal Medicine 2021

$\mathrm{W}$ ith over 30 million infected and almost 600,000 deaths in the USA, the COVID-19 pandemic has underscored the toll of structural inequities. This reality manifests in the disproportionate burden of cases and deaths facing Black Americans, Hispanic Americans, Native Americans, and Pacific Islander Americans. ${ }^{1}$ Amidst heightened racism, ${ }^{2}$ COVID-related health disparities facing the Asian American

Some contents of the paper were previously included in a poster accepted to the Asian Pacific American Medical Student Association (APAMSA) Conference on January 23, 2021.

Received March 11, 2021

Accepted June 24, 2021

Published online August 4, 2021 community remain relatively unknown. In this article, we examine the data on the Asian American COVID-19 death toll using several mortality measures. We consider the relatively lower share of recorded COVID-19 deaths among Asian Americans in the context of growing evidence that shows a high burden of excess deaths, case fatality, and attributable mortality.

\section{POPULATION MORTALITY RATES AND UNCERTAINTY}

A cursory look at population data shows that Asian Americans may have a lower burden of COVID-19 mortality than the overall population. As of March 2, 2021, there were 17,747 Asian American deaths, or $3.6 \%$ of all COVID-19 deaths, compared to a $5.6 \%$ share of the population. ${ }^{1}$ The age-adjusted COVID-19 mortality rate per 100,000 is 117 for Asian Americans compared to 150.2 for the overall population. ${ }^{1}$ However, the population mortality rate may be unreliable due to data collection deficiencies and insufficient testing, especially early in the pandemic. ${ }^{3}$ Thus, considering both confirmed deaths and deaths in excess of a typical year is important to understanding the true extent of mortality from COVID-19.

Recorded COVID-19 deaths may reflect a substantial undercount. Two studies using Centers for Disease Control and Prevention (CDC) data compared all-cause mortality rates in 2020 to prior years. ${ }^{4,5}$ From January to October of 2020, there were $37 \%$ more deaths than usual among Asian Americans, second only to a $53.6 \%$ increase among Hispanic Americans, and three times that of the rise among non-Hispanic Whites $(12 \%){ }^{4}$ Asian Americans had less than half of excess deaths officially linked to COVID-19, substantially lower than the two-thirds attributed to COVID-19 among overall excess deaths. ${ }^{4,5}$ The lower attribution among Asian Americans may reflect a large number of misclassified COVID deaths due to lack of testing or other deficiencies in data. For Asian Americans, the spike in all-cause deaths was most pronounced early in the pandemic between March and April 2020, when testing was most scarce. ${ }^{4}$ The excess deaths could also reflect the indirect effects of COVID-19 such as delayed care for other health conditions. ${ }^{6}$ While the growing size and age of the Asian American population since 2015 would increase the 
number of deaths over time, ${ }^{7}$ the $37 \%$ magnitude of excess deaths cannot be explained by demographic changes alone. Whether through direct or indirect means, the pandemic is the most plausible explanation for the magnitude of excess deaths. "With this new data, Asian Americans join Blacks and Hispanics among the hardest-hit communities," stated authors of the Marshall Project and Associated Press study. ${ }^{5}$

\section{HIGH CASE FATALITY FROM COVID-19}

The first indication of high COVID-19 case fatality among Asian Americans came in May 2020, when a report from San Francisco found that they comprised half of the city's COVID19 deaths despite having accounted for only $13.7 \%$ of cases. ${ }^{8}$ This translated into a three times higher case fatality rate (CFR) than that of non-Hispanic Whites (6.7\% vs. $2.4 \%){ }^{8}$ CFR is the number of COVID-19 deaths divided by the total number of COVID-19 cases and serves as an indicator of disease severity, assuming adequate testing.

A subsequent study revealed a similar pattern of higher CFR for Asian Americans compared to the overall population in many states and counties with larger Asian populations. ${ }^{9}$ For instance, the Asian American CFR was three times the overall ( $8.4 \%$ vs. $2.6 \%$ ) in California, and in Clark County, $\mathrm{NV}$, Asian Americans comprised $17 \%$ of deaths compared to $6.5 \%$ of cases and $10.4 \%$ of the population. ${ }^{9}$

While early studies were limited to specific states and counties and unadjusted, numerous subsequent studies confirmed a higher COVID-19 CFR for Asian Americans. ${ }^{10-16}$ One of the largest studies to date is a Kaiser Family Foundation (KFF) analysis that used EPIC electronic health record (EHR) data of 332,956 COVID-19 patients from 399 hospitals across 21 states. ${ }^{15}$ Compared to the U.S. population, the KFF sample had higher shares of Black and White patients and lower shares of Hispanic and Asian patients, although Asian patients still comprised $3 \%$ of the active patient population and about 11,000 COVID-positive cases. Asian Americans had the highest CFR at $1.5 \%$, compared to $1.0 \%$ for non-Hispanic Whites, $1.2 \%$ for Black Americans, and $1.3 \%$ for Hispanic Americans. Using the CDC's national COVID database, which compiles the cases and deaths recorded by local health departments, the Washington Post analyzed 5.8 million COVID-19 cases reported through October 2020 and found that Asian Americans had a 53\% higher risk of death from COVID-19 infection than non-Hispanic Whites and the highest risk of any group even after adjustment for age, sex, and mortality rates over time. ${ }^{16}$ Findings from an analysis of California COVID cases and deaths were similar. ${ }^{13}$

\section{EXPLANATIONS FOR HIGH CASE FATALITY RATE}

The higher case fatality rate among Asian Americans could be due to an underestimation of positive cases (i.e., low denominator), higher risk of death among positive cases (i.e., high numerator), or more likely both.

\section{Tested Less yet More Likely Positive}

A disproportionate number of COVID-19 cases among Asian Americans may not have been reported or detected. ${ }^{5,15,17}$ Inconsistencies in data reporting have hindered assessment of testing rates by race and ethnicity. ${ }^{9}$ The KFF study was less affected by reporting heterogeniety by using data from a single, large electronic medical record system that included a large sample of Asian Americans. In that study, Asian Americans had the lowest rate of COVID-19 testing of any race or ethnic group at 345 per 10,000 compared to 423 per 10,000 in the overall population. ${ }^{15}$

The lower testing rates were not surprising. Testing sites were less available in minority neighborhoods including those with a high proportion of Asian Americans. ${ }^{18}$ A communitybased survey of nearly 1300 Asian Americans in the San Francisco Bay Area found that only 3\% of Asian Americans had been tested, with $49 \%$ reporting difficulties locating a testing site as reason for not getting tested. ${ }^{19}$ Others have suggested that heightened fears of entering public spaces, the stigma of testing positive, and increased anti-Asian racism might contribute to less testing. ${ }^{20,21}$

Under-testing could manifest as a relatively high positivity rate and reflect a scenario where surveillance (i.e., asymptomatic) testing is disproportionately low in a particular group while those who do get tested are disproportionately symptomatic and thus more likely to test positive. In other words, this might indicate concentrated testing among symptomatic individuals. A large number of studies have found high positivity rates among Asian Americans. ${ }^{14,15,17,22-28}$ They were twice as likely as non-Hispanic White Americans to test positive in the KFF study and second most likely to test positive after Hispanic Americans in the New York City (NYC) Health and Hospitals system. ${ }^{14,15}$ In the University of Washington Medicine system, Vietnamese, Korean, and Mandarin/Cantonese speakers had 4.4, 2.8, and 1.5 times higher positivity rates, respectively, than English speakers. ${ }^{17}$ Together, these studies provide compelling evidence that Asian Americans with COVID-19 have disproportionately gone undiagnosed.

\section{Presenting Sicker and More Likely to Die}

Greater disease severity may also be a factor in the higher CFR. Under the logic that patients with severe COVID-19 are more likely to be admitted to the hospita, 1 hospitalization rates could serve as a proxy for disease severity, and the vast majority of studies show Asian Americans have higher rates of hospitalization. ${ }^{14,15,29-34}$ Three studies in major health systems found 1.5 times higher odds of hospitalization compared to non-Hispanic Whites, similar to the 1.6 times higher risk found in the KFF study, which was the highest of any group. ${ }^{15}$ Furthermore, a retrospective cohort study from 12 hospitals 
and 60 clinics in Minnesota ( $\mathrm{N}=5577$ with $12.2 \%$ Asians) found that Asian Americans had 2.4 times higher odds of hospitalization compared to non-Hispanic Whites; among Asian patients, those with limited-English proficiency had 1.6 times higher odds of hospitalization. ${ }^{34}$ A systematic review and meta-analysis of 50 studies in the USA and UK demonstrated a trend towards higher adjusted relative risk of death (1.22, 95\% confidence interval 0.99-1.50) among Asian patients compared to non-Hispanic White patients. ${ }^{29}$

Among hospitalized patients, two studies in NYC showed Asian Americans dying at lower rates than non-Hispanic Whites, but higher rates than Black and Hispanic Americans. $^{23,30}$ In contrast, a larger study of 88 hospitals in the American Heart Association COVID-19 Cardiovascular Disease Registry found that hospitalized Asian Americans had the highest risk of death of any group. ${ }^{35}$

Delayed presentation to care is another factor. In that same study of 88 hospitals, Asian Americans had on average the highest COVID-19 cardiorespiratory severity at presentation of any group and the longest time from symptom onset to hospital arrival (averaging 7 days). ${ }^{35}$ Late presentation may be a factor in why Asian patients in NYC had the highest rates of critical illness, mechanical ventilation, and ICU admission among all racial/ethnic groups. ${ }^{30}$ In a systematic review of 50 studies, Asian patients were also the only minority group to have a higher risk of admission to intensive care. ${ }^{29}$

Although race/ethnic differences in CFR narrowed after age standardization, ${ }^{12}$ the disparity for Asian Americans cannot be explained by age or other sociodemographic factors alone. ${ }^{15,30,32}$ Importantly, this discussion has focused on national studies or studies in regions of the USA with larger Asian American populations; there is substantial variation in the mortality burden of COVID-19 among Asian Americans in different parts of the country. ${ }^{1,9,12}$

\section{ATTRIBUTABLE MORTALITY}

Another key measure is attributable mortality, or the number of deaths attributed to COVID-19 divided by the number of total deaths. A recent study using data from the National Center for Health Statistics revealed that $13.8 \%$ of deaths among non-Hispanic Asian Americans were attributed to COVID-19, compared to $6.5 \%$ among non-Hispanic Whites and $9.2 \%$ overall. $^{36}$ This means in 2020,1 in 7 deaths in the Asian American community could be attributed to COVID-19 using known COVID-19 deaths alone.

\section{DATA LIMITATIONS}

National data on COVID-19 incidence and mortality for Asian Americans may be inaccurate because many states report limited or no data on this population, and those that do often combine Asian Americans with Pacific Islanders (PI). ${ }^{37}$ Depending on the state or county, Asian American data could be reported under "Asian Americans," "AAPI," "Other," or omitted. Furthermore, "Asian American" encompasses a diverse array of nationalities, ethnic groups, and lived experiences. Lack of data disaggregated by national origin or language group contributes to under-appreciation of disease burden and lack of resources tailored to each group. The COVID-19 pandemic has underscored the significance of data disaggregation. Disaggregating Asian Americans and Pacific Islanders, as the Office of Management and Budget mandates, reveals a disproportionate impact of COVID-19 on Pacific Islanders. ${ }^{38,39}$ Analyzing testing positivity rates by primary language demonstrated a wide range of positivity, ${ }^{17}$ as did disaggregation by national origin for rates of positivity, hospitalization, and mortality. ${ }^{14}$ With disaggregated data from the NYC public hospital system, Chinese patients had the highest mortality rate of any racial or ethnic group, while South Asians had the highest rates of positivity and hospitalization among Asian subgroups. ${ }^{14}$ Filipino Americans have also experienced disproportionate mortality in California. ${ }^{40}$

A further issue is the continued omission of Asian Americans and their subgroups in the scientific literature. During our review, we observed numerous studies, including those published in the highest impact journals, on health disparities with no mention of Asian Americans.

\section{IMPLICATIONS OF RACISM}

Recent attacks on Asian Americans, particularly the elderly, across the country have called attention to the persistent antiAsian racism that has been exacerbated by the COVID-19 pandemic. The omission of Asian Americans from discussion of health disparities is itself a form of racism that has serious consequences. For instance, the National Academy of Sciences, Medicine, and Engineering excluded Asian Americans from their list of groups experiencing disproportionate impact from COVID-19 and did not mention Asian Americans in their "Framework for Equitable Allocation of COVID-19 Vaccine. ${ }^{, 41}$ Other factors contributing to the exclusion of Asian Americans from analyses include failure to recruit enough Asian Americans for studies, language barriers in participant recruitment and research design, limited funding towards AAPI research, concerns related to immigration status, and mistrust in the research process among Asian American communities. ${ }^{42,43}$

Biases that stem from the "Model Minority" myth may contribute to the high mortality burden on Asian Americans. The model minority myth, in which Asian Americans are assumed to be doing better than other minority groups, was created in the 1960s to challenge the coalition of people of color during the Civil Rights movement. The racism underlying the "Model Minority" myth harms Asian Americans by perpetuating the perception that they do not have disparities and therefore are unworthy of resources, which leads to lack of data or inaccurate data for this population that then reinforces 
the misperception that Asian Americans do not have disparities. In the COVID pandemic, this manifests in the use of inadequate data to exclude Asian Americans as a group with disparities. For Asian Americans, the absence of evidence is often not the evidence of absence, but the evidence of neglect due to racism.

When it comes to COVID-19 vaccinations, Asian Americans have rates of vaccination similar to that of non-Hispanic Whites. ${ }^{44}$ This may be due in part to the relatively high share of Asian Americans in the essential healthcare workforce, who were first eligible for vaccines. ${ }^{20}$ Despite the promising vaccination rates in the aggregate, vulnerable Asian Americans including low-income individuals and the elderly face additional barriers to accessing vaccines that clinicians and policymakers should not overlook. ${ }^{45}$

\section{CONCLUSIONS}

Asian Americans experience significant COVID-19 disparities, including a higher attributable mortality burden than non-Hispanic Whites. The evidence indicate that Asian Americans are under-tested for COVID-19 and experience higher risk of complications and death from COVID-19. The role of historical and present-day racism in both overt and covert forms cannot be understated in understanding why COVID19 disparities among Asian Americans receive little acknowledgement. Nonetheless, the history of Asian Americans, and indeed all communities of color in America, is one of resilience and hope in progress. For Asian Americans, it begins with recognizing diverse experiences and challenges, disaggregating data by subgroup, and unifying efforts against the simultaneous omission and heightened racism they endure in the face of a historic pandemic.

Corresponding Author: Brandon W. Yan, B.A.; School of Medicine, University of California San Francisco, 513 Parnassus Ave., Suite S-214, San Francisco, CA 94143, USA (e-mail: Brandon.Yan@ucsf. edu).

\section{Declarations:}

There were no contributors that did not meet the criteria for authorship.

Conflict of Interest: The authors declare that they do not have a conflict of interest.

\section{REFERENCES}

1. APM Research Lab Staff. The color of coronavirus: COVID-19 deaths by race and ethnicity in the U.S. APM Research Lab. Published March 5, 2021. Accessed May 17, 2021. https://www.apmresearchlab.org/covid/ deaths-by-race

2. Combatting racism against Asian Americans and Pacific Islanders. UNESCO. Published November 10, 2020. Accessed December 22, 2020. https://en.unesco.org/news/combatting-racism-against-asian-americans-and-pacific-islanders
3. Bruer W, Simko-Bednarski E, Azad A, Hilk M. In strategic shift, doctors in America's two largest cities are told to skip some coronavirus testing. CNN. Published March 21, 2020. Accessed January 1, 2021. https:// www.cnn.com/world/live-news/coronavirus-outbreak-03-21-20-intlhnk/index.html

4. Rossen LM, Branum A, Ahmad F, Sutton P, Anderson R. Excess Deaths Associated with COVID-19, by Age and Race and Ethnicity United States, January 26-October 3, 2020. MMWR Morb Mortal Wkly Rep. 2020;69(42):1522-1527. doi:https://doi.org/10.15585/mmwr. mm6942e2

5. Flagg A, Sharma D, Fenn L, Stobbe M. COVID-19's Toll on People of Color Is Worse Than We Knew. The Marshall Project. Published August 21, 2020. Accessed December 20, 2020. https://www. themarshallproject.org/2020/08/21/covid-19-s-toll-on-people-of-coloris-worse-than-we-knew

6. Czeisler MÉ. Delay or Avoidance of Medical Care Because of COVID19-Related Concerns - United States, June 2020. MMWR Morb Mortal Wkly Rep. 2020;69. doi:https://doi.org/10.15585/mmwr. mm6936a4

7. Multiple Cause of Death 1999-2019 on CDC WONDER Online Database. Centers for Disease Control and Prevention, National Center for Health Statistics. Published 2020. Accessed April 26, 2021. https://wonder.cdc. gov/controller/saved/D77/D151F895

8. Yan B, Ng F, Nguyen T. High Mortality from COVID-19 among Asian Americans in San Francisco and California. Asian American Research Center on Health; 2020. Accessed May 13, 2020. https://asianarch.org/ press_releases/Asian\%20COVID-19\%20Mortality\%20Final.pdf

9. Yan BW, Ng F, Chu J, Tsoh J, Nguyen T. Asian Americans Facing High COVID-19 Case Fatality. Health Affairs Blog. Published July 13, 2020. Accessed July 13, 2020. https://www.healthaffairs.org/do/10.1377/ hblog20200708.894552/full/

10. Artiga S, Corallo B, Pham O. Racial Disparities in COVID-19: Key Findings from Available Data and Analysis. KFF. Published August 17, 2020. Accessed December 21, 2020. https://www.kff.org/report-section/ racial-disparities-in-covid-19-key-findings-from-available-data-and-analysis-issue-brief/

11. Renelus BD, Khoury NC, Chandrasekaran K, et al. Racial Disparities in COVID-19 Hospitalization and In-hospital Mortality at the Height of the New York City Pandemic. J Racial Ethn Health Disparities. Published online September 18, 2020:1-7. doi:https://doi.org/10.1007/s40615020-00872-X

12. Pathak I, Choi Y, Jiao D, Yeung D, Liu L. Racial-ethnic disparities in case fatality ratio narrowed after age standardization: A call for raceethnicity-specific age distributions in State COVID-19 data. medRxiv. Published online October 4, 2020:2020.10.01.20205377. doi:https:// doi.org/10.1101/2020.10.01.20205377

13. Xiong $\mathbf{D}$, Zhang $\mathbf{L}$, Watson GL, et al. Pseudo-likelihood based logistic regression for estimating COVID-19 infection and case fatality rates by gender, race, and age in California. Epidemics. 2020;33:100418. doi:https://doi.org/10.1016/j.epidem.2020.100418

14. Marcello RK, Dolle J, Tariq A, et al. Disaggregating Asian Race Reveals COVID-19 Disparities among Asian Americans at New York City's Public Hospital System. medRxiv. Published online November 24, 2020:2020.11.23.20233155. doi:https://doi.org/10.1101/2020.11.23. 20233155

15. Rubin-Miller L, Alban C, Artiga S, Sullivan S. COVID-19 Racial Disparities in Testing, Infection, Hospitalization, and Death: Analysis of Epic Patient Data. KFF. Published September 16, 2020. Accessed December 20, 2020. https://www.kff.org/coronavirus-covid-19/issuebrief/covid-19-racial-disparities-testing-infection-hospitalization-deathanalysis-epic-patient-data/

16. Keating D, Cha AE, Florit G. 'I just pray God will help me': Racial, ethnic minorities reel from higher covid-19 death rates. Washington Post. Published November 20, 2020. Accessed January 11, 2021. https:// www.washingtonpost.com/graphics/2020/health/covid-race-mortalityrate/

17. Kim HN, Lan KF, Nkyekyer E, et al. Assessment of Disparities in COVID-19 Testing and Infection Across Language Groups in Seattle, Washington. JAMA Netw Open. 2020;3(9):e2021213. doi:https://doi.org/ 10.1001/jamanetworkopen.2020.21213

18. Kim SR, Vann M, Bronner L, Manthey G. Which Cities Have The Biggest Racial Gaps In COVID-19 Testing Access? FiveThirtyEight. Published July 22, 2020. Accessed December 23, 2020. https://fivethirtyeight. $\mathrm{com} /$ features/white-neighborhoods-have-more-access-to-covid-19-testing-sites / 
19. Guach T, Doan L, Liou J, Ponce N. Simultaneously Blamed and Ignored: Barriers, Behaviors, and Impact of COVID-19 on Asian Americans. Published online August 30, 2020. Accessed December 23, 2020. https://preprints.jmir.org/preprint/23976

20. AAPIs Rising to Fight Dual Pandemics Covid-19 and Racism. One Nation Commission; 2020. Accessed December 23, 2020. https://www. onenationaapi.com/commission-report-ii

21. Marco della Cava. Asian Americans in San Francisco are dying at alarming rates from COVID-19: Racism is to blame. USA Today. Published October 21, 2020. Accessed December 20, 2020. https:// www.usatoday.com/in-depth/news/nation/2020/10/18/coronavirusasian-americans-racism-death-rates-san-francisco/5799617002/

22. Rozenfeld Y, Beam J, Maier $\mathbf{H}$, et al. A model of disparities: risk factors associated with COVID-19 infection. Int $J$ Equity Health. 2020;19(1): 126. doi:https://doi.org/10.1186/s12939-020-01242-z

23. Kabarriti R, Brodin NP, Maron MI, et al. Association of Race and Ethnicity With Comorbidities and Survival Among Patients With COVID19 at an Urban Medical Center in New York. JAMA Netw Open. 2020;3(9):e2019795. doi:https://doi.org/10.1001/jamanetworkopen. 2020.19795

24. Mackey K, Ayers CK, Kondo KK, et al. Racial and Ethnic Disparities in COVID-19-Related Infections, Hospitalizations, and Deaths : A Systematic Review. Ann Intern Med. Published online December 1, 2020. doi:https://doi.org/10.7326/M20-6306

25. Abedi V, Olulana O, Avula V, et al. Racial, Economic, and Health Inequality and COVID-19 Infection in the United States. Springer Link. Published September 1, 2020. Accessed December 21, 2020. https:// link.springer.com/article/10.1007/s40615-020-00833-4

26. Vahidy FS, Nicolas JC, Meeks JR, et al. Racial and Ethnic Disparities in SARS-CoV-2 Pandemic: Analysis of a COVID-19 Observational Registry for a Diverse U.S. Metropolitan Population. medRxiv. Published online May 12, 2020:2020.04.24.20073148. doi:https://doi.org/10.1101/ 2020.04.24.20073148

27. Shah SJ, Barish PN, Prasad PA, et al. Clinical features, diagnostics, and outcomes of patients presenting with acute respiratory illness: a comparison of patients with and without COVID-19. medRxiv. Published online May 6, 2020:2020.05.02.20082461. doi:https://doi.org/10.1101/ 2020.05.02.20082461

28. Rosenberg ES, Tesoriero JM, Rosenthal EM, et al. Cumulative incidence and diagnosis of SARS-CoV-2 infection in New York. medRxiv. Published online May 29, 2020:2020.05.25.20113050. doi:https://doi. org/10.1101/2020.05.25.20113050

29. Sze S, Pan D, Nevill CR, et al. Ethnicity and clinical outcomes in COVID-19: A systematic review and meta-analysis. EClinicalMedicine. 2020;29. doi:https://doi.org/10.1016/j.eclinm.2020.100630

30. Ogedegbe G, Ravenell J, Adhikari S, et al. Assessment of Racial/Ethnic Disparities in Hospitalization and Mortality in Patients With COVID-19 in New York City. JAMA Netw Open. 2020;3(12):e2026881. doi:https://doi. org/10.1001/jamanetworkopen.2020.26881

31. Petrilli CM, Jones SA, Yang J, et al. Factors associated with hospitalization and critical illness among 4,103 patients with Covid-19 disease in New York City. medRxiv. Published online April 11 , 2020:2020.04.08.20057794. doi:https://doi.org/10.1101/2020.04.08. 20057794

32. Dai C, Kornilov S, Roper R, et al. Characteristics and Factors Associated with COVID-19 Infection, Hospitalization, and Mortality Across Race and Ethnicity. Social Science Research Network; 2020. doi:https://doi.org/ $10.2139 /$ ssrn.3712938
33. Azar KMJ, Shen Z, Romanelli RJ, et al. Disparities In Outcomes Among COVID-19 Patients In A Large Health Care System In California. Health Affairs. 2020;39(7):1253-1262. doi:https://doi.org/10.1377/hlthaff. 2020.00598

34. Ingraham NE, Purcell LN, Karam BS, et al. Racial and Ethnic Disparities in Hospital Admissions from COVID-19: Determining the Impact of Neighborhood Deprivation and Primary Language. $J$ GEN INTERN MED. Published online May 18, 2021. doi:https://doi.org/10. 1007/s11606-021-06790-w

35. Rodriguez Fatima, Solomon Nicole, de Lemos James A., et al. Racial and Ethnic Differences in Presentation and Outcomes for Patients Hospitalized with COVID-19: Findings from the American Heart Association's COVID-19 Cardiovascular Disease Registry. Circulation. 2020;0(0). doi:https://doi.org/10.1161/CIRCULATIONAHA.120.052278

36. Chu JN, Tsoh JY, Ong E, Ponce NA. The Hidden Colors of Coronavirus: the Burden of Attributable COVID-19 Deaths. J Gen Intern Med. Published online January 22, 2021:1-3. doi:https://doi.org/10.1007/ s11606-020-06497-4

37. National Council of Asian Pacific Islander Physicians. NCAPIP Statement on COVID-19 Disparities. Published online June 9, 2020. Accessed December 23, 2020. https://waysandmeans.house.gov/sites/democrats.waysandmeans.house.gov/files / d ocuments / NCAPIP\%20Statement\%20on\%20COVID-19\%20Disparities.pdf

38. Wang D, Gee GC, Bahiru E, Yang EH, Hsu JJ. Asian-Americans and Pacific Islanders in COVID-19: Emerging Disparities Amid Discrimination. J Gen Intern Med. Published online October 2, 2020:1-4. doi:https://doi.org/10.1007/s11606-020-06264-5

39. Chang RC, Penaia C, Thomas K. Count Native Hawaiian And Pacific Islanders In COVID-19 Data-It's An OMB Mandate | Health Affairs Blog. Health Affairs Blog. Published August 27, 2020. Accessed December 23, 2020. https://doi.org/10.1377/hblog20200825.671245/full/

40. Wong $\mathbf{T}$. Little noticed, Filipino Americans are dying of COVID-19 at an alarming rate. Los Angeles Times. https://www.latimes.com/california/ story/2020-07-21/filipino-americans-dying-covid. Published July 21, 2020. Accessed December 23, 2020.

41. Framework for Equitable Allocation of COVID-19 Vaccine. The National Academies Press; 2020. doi:https://doi.org/10.17226/25917

42. George S, Duran N, Norris K. A Systematic Review of Barriers and Facilitators to Minority Research Participation Among African Americans, Latinos, Asian Americans, and Pacific Islanders. Am J Public Health. 2013;104(2):e16-e31. doi:https://doi.org/10.2105/AJPH.2013.301706

43. Đoàn LN, Takata Y, Sakuma K-LK, Irvin VL. Trends in Clinical Research Including Asian American, Native Hawaiian, and Pacific Islander Participants Funded by the US National Institutes of Health, 1992 to 2018. JAMA Netw Open. 2019;2(7). doi:https://doi.org/10.1001/ jamanetworkopen.2019.7432

44. COVID Data Tracker. Centers for Disease Control and Prevention. Published May 25, 2021. Accessed May 26, 2021. https://covid.cdc. gov/covid-data-tracker

45. Tseng A. Not all Asian Americans are being vaccinated at high rates. A Chinatown clinic shows why. Los Angeles Times. Published April 21, 2021. Accessed April 26, 2021. https://www.latimes.com/california/ story/2021-04-21/chinatown-vaccine-clinic-asian-american-seniors

Publisher's Note: Springer Nature remains neutral with regard to jurisdictional claims in published maps and institutional affiliations. 